\title{
Effect of episodic accretion on the structure and the lithium depletion of low-mass stars and planet-hosting stars
}

\author{
I. Baraffe $e^{1,2}$ and G. Chabrier ${ }^{2,1}$ \\ ${ }^{1}$ School of Physics, University of Exeter Stocker, Road, Exeter, EX4 4QL, UK \\ e-mail: ibaraffe, chabrier@ens-lyon.fr \\ 2 École Normale Supérieure, Lyon, CRAL (UMR CNRS 5574), Université de Lyon, France
}

Received 11 May 2010 / Accepted 15 July 2010

\begin{abstract}
Following up our recent analysis devoted to the impact of non steady accretion on the location of young low-mass stars or brown dwarfs in the Herzsprung-Russell diagram, we perform a detailed analysis devoted to the effect of burst accretion on the internal structure of low-mass and solar type stars. We find that episodic accretion can produce objects with significantly higher central temperatures than the ones of the non accreting counterparts of same mass and age. As a consequence, lithium depletion can be severely enhanced in these objects. This provides a natural explanation for the unexpected level of lithium depletion observed in young objects for the inferred age of their parent cluster. These results confirm the limited reliability of lithium abundance as a criterion for assessing or rejecting cluster membership. They also show that lithium is not a reliable age indicator, because its fate strongly depends on the past accretion history of the star. Under the assumption that giant planets primarily form in massive disks prone to gravitational instability and thus to accretion burst episodes, the same analysis also explains the higher Li depletion observed in planet hosting stars. At last, we show that, depending on the burst rate and intensity, accretion outbursts can produce solar mass stars with lower convective envelope masses, at ages less than a few tens of Myr, than predicted by standard (non or slowly accreting) pre-main sequence models. This result has interesting, although speculative, implications for the recently discovered depletion of refractory elements in the Sun.
\end{abstract}

Key words. stars: formation - stars: low-mass - stars: abundances - accretion, accretion disks

\section{Introduction}

There is a growing consensus in the star formation community that non steady (episodic) accretion plays a dominant role during the formation of low-mass stars (see e.g. Enoch et al. 2009; Vorobyov 2009; Zhu et al. 2010a, and references therein). In a recent paper (Baraffe et al. 2009, hereafter BCG09), we have suggested that episodic accretion provides a viable explanation for the observed luminosity spread in young cluster HerzsprungRussell diagrams (HRD). The present follow-up analysis explores in more details the effects of episodic accretion on the internal structure of young low mass stars $\left(\leq 1 M_{\odot}\right)$, commonly used to derive ages of star forming regions and young clusters. We show that, depending on the accretion history, the internal structure of these objects can be strongly affected for up to a few tens of Myr (Sect. 2). Lithium depletion and, for the partly convective stars, the size of the convective envelope, in particular can strongly differ from the standard (non accreting) pre-main sequence model predictions (Sect. 3). In Sect. 4, we examine the impact of episodic accretion on the observational signatures and show that taking this process into account in the young lowmass object evolution provides a consistent explanation for the puzzling observations of strong lithium depletion in several lowmass stars (LMS) belonging to young clusters (e.g. Kenyon et al. 2005; Sacco et al. 2007) and in planet-hosting stars (Israelian et al. 2009), as well as to the recently determined peculiar abundances of refractory elements in the Sun (Melendez et al. 2009; Ramirez et al. 2009).

\section{Effect of mass accretion on the internal structure}

\subsection{Evolutionary models with accretion}

We adopt the same input physics and the same treatment of accretion as outlined in BCG09. In standard stellar evolution calculations, energy conservation equation for a non accreting object reads as

$\left(\frac{\partial L}{\partial m}\right)_{t}=-T\left(\frac{\partial S}{\partial t}\right)_{m}+\epsilon_{\mathrm{nuc}}$,

where $m$ is the mass enclosed in a sphere of radius $r$ within the object, $S$ the specific entropy and $\epsilon_{\text {nuc }}$ the local nuclear energy generation rate. For very young objects, only deuterium fusion provides a contribution to $\epsilon_{\text {nuc }}{ }^{1}$. For accretion proceeding at a rate $\dot{M}$, time derivatives at fixed mass shell must account for the variation of mass with time and of entropy with accreted mass, i.e. (Sugimoto \& Nomoto 1975):

$\left(\frac{\partial S}{\partial t}\right)_{m}=\left(\frac{\partial S}{\partial t}\right)_{q}-\dot{m}\left(\frac{\partial S}{\partial m}\right)_{t}$

with $q \equiv m / M_{*}$. The mass $(\dot{M} \cdot \Delta t)$ accreted during a timestep $\Delta t$ is assumed to be redistributed over the entire structure, with the new mass in a shell of fixed $q$ given by

$m(t+\Delta t)=q \times\left(M_{*}(t)+\dot{M} \Delta t\right)$.

1 The nuclear energy generation produced by lithium fusion is completely negligible. 
In the present calculations, we assume instantaneous and uniform redistribution of the extra source of internal energy brought by the accreted material. In reality, mass and heat redistributions inside the accreting object depend on the thermal properties of the accreting material. Proto low-mass stars below about $2 M_{\odot}$ are expected to be entirely convective (Stahler \& Palla 2005). For completely convective objects, given the short typical convective timescales, our assumption of uniform mass and heat redistribution should be valid, providing the entropy of accreted matter is comparable to or less than the accreting object's internal one, so that the infalling material can rapidly thermalize with its surroundings (see discussion in Siess \& Forestini 1996; Hartmann et al. 1997). If the aforementioned condition is not fulfilled, the accreting matter may not be able to penetrate very deep inside the object, possibly leading to Rayleigh-Taylor like instabilities in stably radiative regions. Siess et al. (1997) explored the effect of mass and heat redistribution by using a penetration function. Such an approach, however, remains highly phenomenological and thus of limited reliability. Given the complexity of the problem and the present exploratory nature of the effect of episodic accretion on young low-mass objects, not mentioning uncertainties in the accretion processes, we elected to stick to the simplest and probably most reasonable in most of the presently explored situations treatment, and assumed homogeneous, rapid heat and matter redistribution within the accreting body.

Omitting the fraction $\epsilon(1-\alpha) \frac{G M M}{R}$ of the accretion shock energy radiated away (see Eqs. (1) and (2) of BCG09 for the definition of $\alpha$ and $\epsilon$ ), which does not affect the accreting object's structure and evolution, the intrinsic luminosity of the protostar or brown dwarf is given by

$L_{*}=\alpha \epsilon \frac{G M \dot{M}}{R}+\int_{M} \epsilon_{\mathrm{nuc}} \mathrm{d} m-\int_{M} T\left\{\left(\frac{\partial S}{\partial t}\right)_{q}-\dot{m}\left(\frac{\partial S}{\partial m}\right)_{t}\right\} \mathrm{d} m$. (4)

As mentioned above, we assume in the present calculations that the accreted matter reaches the stellar surface with a lower specific entropy than the object's internal one, which implies $\alpha \rightarrow 0$ in Eq. (4). As shown in BCG09, calculations based on this assumption provide a consistent explanation of the luminosity spread in young cluster HRDs, while high values of $\alpha$ would predict overluminous objects during a short period of time (because of the short corresponding Kelvin-Helmholtz timescale, see discussion in BCG09). Addition of fresh infalling deuterium and lithium is accounted for during the accretion process, with interstellar mass fractions $[\mathrm{D}]_{0}=2 \times 10^{-5}$ and $[\mathrm{Li}]_{0}=10^{-9}$, respectively. Depending on the accretion rate, these elements can start to be consumed at early stages in the proto-object interior during the burst accretion phases. The implementation of the accretion process in the stellar evolution code has been validated (Gallardo 2007; Gallardo et al. 2009) by comparing our numerical results to those derived from the polytropic approach of Stahler (1988) and Hartmann et al. (1997) in the limit of validity of the polytropic model, i.e. for initial masses $\gtrsim 0.1 M_{\odot}$.

\subsection{Effect of episodic accretion on the internal structure}

As in BCG09, our accretion histories are based on the calculations of Vorobyov \& Basu (2005), obtained from gravitational instability in the accreting centrifugally supported disk. Starting from arbitrary initial masses $M_{\text {init }}$, we vary the number of bursts $N_{\text {burst }}$, with accretion rate $\dot{M}_{\text {burst }}$ and duration $\Delta t_{\text {burst }}$, separated by quiescent phases of duration $\Delta t_{\text {quiet }}$ and accretion rate $\dot{M}_{\text {quiet }}$. We explored a wide range of parameters, inferred from the results of Vorobyov \& Basu (2005), with the aim of obtaining final LMSs within the mass range $0.1-1 M_{\odot}$, in order to focus on the typical population used to derive the ages of star-forming regions and young clusters. Brown dwarf cases have been shown in BCG09. Our initial masses $M_{\text {init }}$ range from $1 M_{\text {Jup }}$ to $0.2 M_{\odot}$. The typical burst number, accretion rates, and duration lie in the ranges $N_{\text {burst }}=2-30, \dot{M}_{\text {burst }}=10^{-4}-5 \times 10^{-4} M_{\odot} \mathrm{yr}^{-1}$, and $\Delta t_{\text {burst }}=$ 100-500 yr, respectively, while quiescent phases last between $\Delta t_{\text {quiet }}=10^{3}$ and $10^{4} \mathrm{yr}$ with accretion rates $\dot{M}<10^{-6} M_{\odot} \mathrm{yr}^{-1}$. As mentioned in BCG09, accretion rates during the quiescent phase below this value have no significant impact on the final internal structure of the accreting object. For the sake of simplicity, we have thus adopted $\dot{M}_{\text {quiet }}=0$ in the present analysis, however, this accretion luminosity must be taken into account when comparing the final luminosity with observations of protoobjects during the embedded phase. The next two sections illustrate the typical results for two examples at each end of the presently studied mass range.

\subsubsection{Sequences leading to the formation of a $0.1 M_{\odot}$ star}

Various evolutionary sequences of episodic accretion, with different initial masses, leading to a final $0.1 M_{\odot}$ LMS are portrayed in Fig. 1. As mentioned in BCG09, high accretion rates yield significantly more compact structures, i.e., smaller radii compared to those of non accreting objects of same mass and age. This contraction comes from the increase in gravitational energy as mass is added, yielding higher central pressures and temperatures compared with the non accreting case. If the accretion timescale, $\tau_{\dot{M}}=M_{*} / \dot{M}$, is higher than the Kelvin-Helmholtz timescale, $\tau_{\mathrm{KH}}=G M_{*}^{2} /\left(R_{*} L_{*}\right)$, the object can relax to the radius it would have in the absence of accretion. In contrast, if $\tau_{\dot{M}} \leq \tau_{\mathrm{KH}}$, which is the case for $\dot{M}_{\text {burst }}=10^{-4}-5 \times 10^{-4} M_{\odot} \mathrm{yr}^{-1}$, the structure has no time to adjust to the incoming mass and energy, and the radius remains smaller than the non accreting counterpart of same mass and age, as illustrated in Fig. 1. The more compact structure of the accreting object yields higher central temperature compared to the non accreting counterpart. Figure 1 highlights the main effects of episodic accretion on proto lowmass objects, which can be summarized as follows.

- For a given initial mass $M_{\text {init }}$, the higher $\dot{M}_{\text {burst }}$ during the burst phases, the smaller the radius of the object at the end of the accretion phase, hence the higher its central temperature. This can be seen by comparing the long-dashed blue curve $\left(M_{\text {init }}=1 M_{\text {Jup }}, \dot{M}_{\text {burst }}=5 \times 10^{-4} M_{\odot} \mathrm{yr}^{-1}\right)$ and the dash-dotted magenta curve $\left(M_{\text {init }}=1 M_{\text {Jup }}, \dot{M}_{\text {burst }}=\right.$ $10^{-4} M_{\odot} \mathrm{yr}^{-1}$ ).

- For a given burst accretion rate $\dot{M}_{\text {burst }}$, the lower the initial mass $M_{\text {init }}$, the greater the impact on the internal structure, i.e., the smaller the radius and the higher the central temperature at the end of the accretion history. This is illustrated by the comparison between the dash-dotted magenta curve $\left(M_{\text {init }}=1 M_{\text {Jup }}, \dot{M}_{\text {burst }}=10^{-4} M_{\odot} \mathrm{yr}^{-1}\right)$ with the dotted red curve $\left(M_{\text {init }}=10 M_{\text {Jup }}, \dot{M}_{\text {burst }}=10^{-4} M_{\odot} \mathrm{yr}^{-1}\right)$.

It is worth stressing that the sequences starting with $M_{\text {init }}=$ $1 M_{\text {Jup }}$ in Fig. 1 reach high enough central temperatures during the burst accretion episodes to completely burn deuterium, including the extra accreted fresh one. This extra supply of nuclear energy is not sufficient to balance the increase in gravitational energy, hence the contraction of the object induced by the added matter. Deuterium burning starts when the central temperature $T_{\mathrm{c}}$ reaches $\sim 10^{6} \mathrm{~K}$. For the two sequences starting with $M_{\text {init }}=1 M_{\text {Jup }}$ and $\dot{M}_{\text {burst }}=10^{-4} M_{\odot} \mathrm{yr}^{-1}$ 

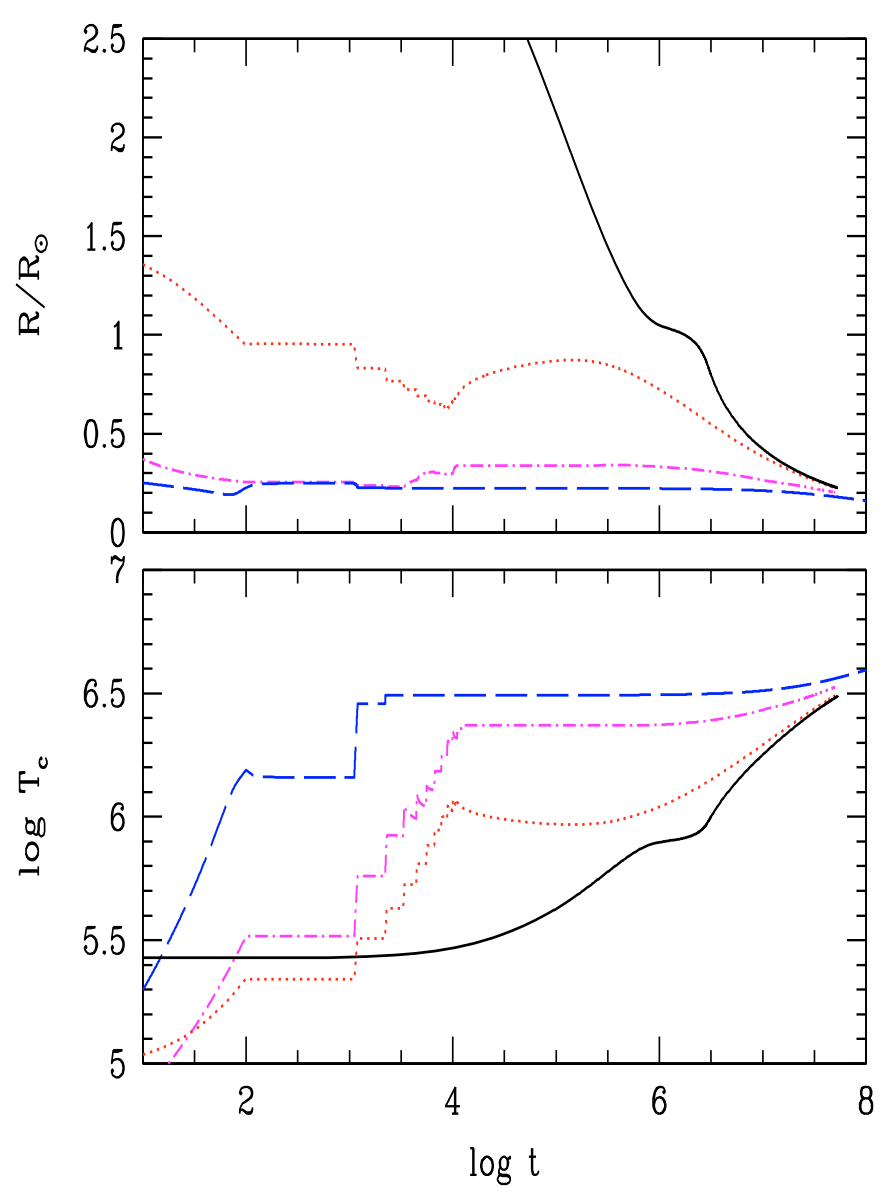

Fig. 1. Evolution of the radius (upper panel) and of the central temperature (lower panel) as a function of time (in yr) for models with episodic accretion and reaching a final mass of $0.1 M_{\odot}$ (see Sect. 2.2.1). Longdash (blue): $M_{\text {init }}=1 M_{\text {Jup }}, \dot{M}_{\text {burst }}=510^{-4} M_{\odot} \mathrm{yr}^{-1}, N_{\text {burst }}=2$; dash-dot (magenta): $M_{\text {init }}=1 M_{\text {Jup }}, \dot{M}_{\text {burst }}=10^{-4} M_{\odot} \mathrm{yr}^{-1}, N_{\text {burst }}=10$; dot (red): $M_{\text {init }}=10 M_{\text {Jup }}, \dot{M}_{\text {burst }}=10^{-4} M_{\odot} \mathrm{yr}^{-1}, N_{\text {burst }}=9$. All calculations are done with $\Delta t_{\text {burst }}=100 \mathrm{yr}$ and $\Delta t_{\text {quiet }}=1000 \mathrm{yr}$. The solid line (black) corresponds to the evolution of a non accreting $0.1 M_{\odot}$ star.

and $\dot{M}_{\text {burst }}=5 \times 10^{-4} M_{\odot} \mathrm{yr}^{-1}$, this corresponds to central densities of $\rho_{\mathrm{c}}=20$ and $30 \mathrm{~g} \mathrm{~cm}^{-3}$, respectively. This stage is reached when both sequences reach a mass of $\sim 0.03 M_{\odot}$. For the sequence starting with $M_{\text {init }}=10 M_{\text {Jup }}$ and $\dot{M}_{\text {burst }}=10^{-4} M_{\odot} \mathrm{yr}^{-1}$, the same central temperature is reached near the end of the accretion phase, for a central density of $\sim 2 \mathrm{~g} \mathrm{~cm}^{-3}$ and a total mass $\sim 0.08 M_{\odot}$. For this sequence, a significant fraction of deuterium ( $\sim 65 \%$ of the initial mass fraction in the fully convective interior) is still present at the end of the accretion phase, generating an important source of nuclear energy which produces the increase in $R$ and then decrease in $T_{\mathrm{c}}$ between $10^{4} \mathrm{yr}$ and $10^{5} \mathrm{yr}$, as shown in Fig. 1.

Finally, Fig. 1 clearly illustrates, for the sequences starting with $1 M_{\text {Jup }}$, the strong differences in radius and central temperature after a few Myr, up to $30 \mathrm{Myr}$, between a $0.1 M_{\odot}$ star produced by episodic accretion with $\dot{M}_{\text {burst }}>10^{-4} M_{\odot} \mathrm{yr}^{-1}$ and the non accreting counterpart. We also stress that, for initial masses $M_{\text {init }} \gtrsim 0.05 M_{\odot}$ and burst accretion rates $\dot{M}_{\text {burst }}<$ $5 \times 10^{-4} M_{\odot} \mathrm{yr}^{-1}$, accretion history hardly affects the internal structure and location in the HRD after $\sim 1$ Myr, producing objects of $0.1 M_{\odot}$ with properties similar to those predicted by standard (non accreting) pre-main sequence models at ages $\geq 1$ Myr.
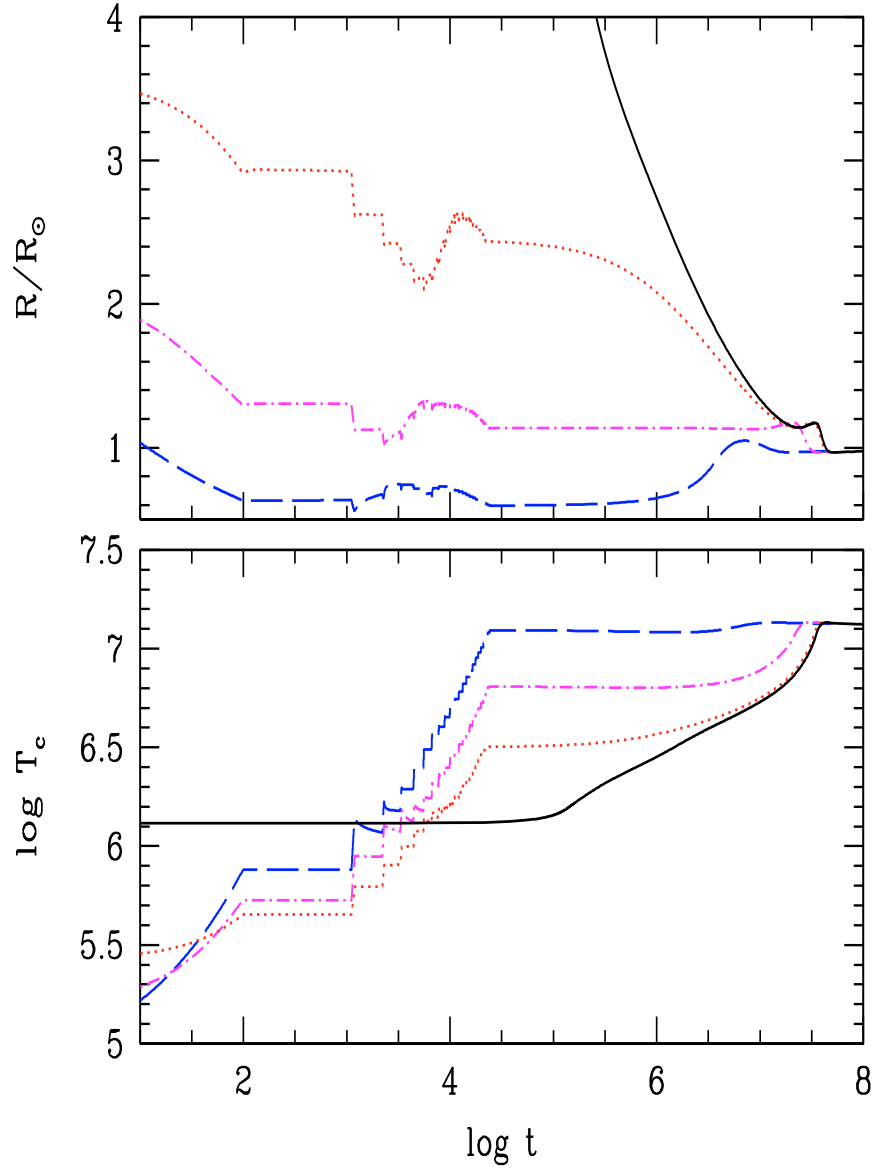

Fig. 2. Same as Fig. 1 for models reaching a final mass $1 M_{\odot}$ (see Sect. 2.2.2). Long-dash (blue): $M_{\text {init }}=10 M_{\text {Jup }}, N_{\text {burst }}=20$; dash-dot (magenta): $M_{\text {init }}=30 M_{\text {Jup }}, N_{\text {burst }}=20 ; \operatorname{dot}\left(\right.$ red): $M_{\text {init }}=0.1 M_{\odot}$, $N_{\text {burst }}=18$. All calculations are done with $\dot{M}_{\text {burst }}=5 \times 10^{-4} M_{\odot} \mathrm{yr}^{-1}$, $\Delta t_{\text {burst }}=100 \mathrm{yr}$ and $\Delta t_{\text {quiet }}=1000 \mathrm{yr}$. The solid line (black) corresponds to the evolution of a non accreting $1 M_{\odot}$ star.

\subsubsection{Sequences leading to the formation of a $1 M_{\odot}$ star}

Figure 2 displays the same analysis for episodic accretion sequences producing $1 M_{\odot}$ objects. The figure portrays the results with $\dot{M}_{\text {burst }}=5 \times 10^{-4} M_{\odot} \mathrm{yr}^{-1}$, which yields the strongest and most interesting effects on the structure and on lithium depletion (see Sect. 3). As found previously, the lower the initial mass, the greater the effect on the structure for a given accretion rate. For initial masses $M_{\text {init }}<0.1 M_{\odot}$ and such high burst accretion rates, the figure illustrates the strong departure in radius and central temperature of these models from the non accreting sequence up to 20-30 Myr. For higher initial masses and $\dot{M}_{\text {burst }}<5 \times 10^{-4} M_{\odot} \mathrm{yr}^{-1}$, the accretion history is found not to significantly alter the properties of the newly formed $1 M_{\odot}$ star, producing an object with a position in the HRD and internal structure similar to the predictions of a non accreting evolutionary sequence at ages $\geq 1$ Myr.

\section{Effect on the size of the convective envelope and on lithium depletion}

\subsection{The case of fully convective stars}

The significantly higher central temperatures induced by episodic accretion affect the timescale of lithium depletion, leading to faster lithium depletion compared with a non accreting 


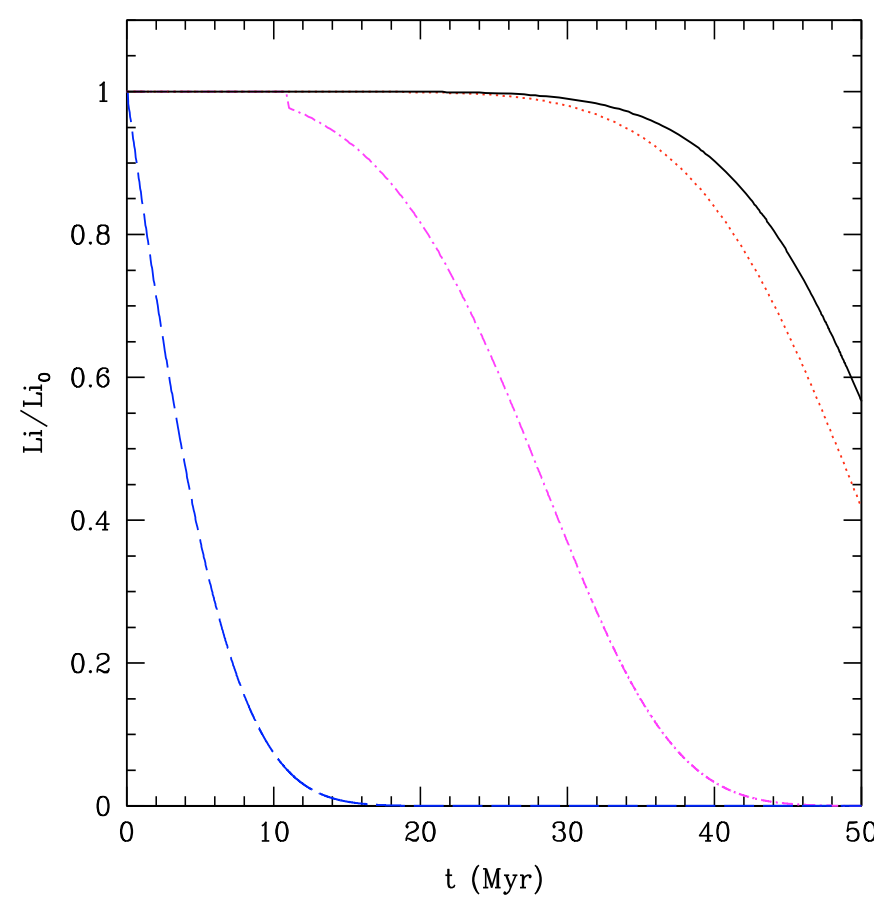

Fig. 3. Evolution of the lithium abundance (divided by the initial $\mathrm{Li}$ abundance) as a function of time for the same models producing a $0.1 M_{\odot}$ low-mass star as in Fig. 1 .

object of the same age, as illustrated in Fig. 3 for sequences producing $0.1 M_{\odot}$ stars. Initial $M_{\text {init }}=1 M_{\text {Jup }}$ protostars experiencing burst accretion rates $\dot{M}_{\text {burst }}=5 \times 10^{-4} M_{\odot} \mathrm{yr}^{-1}$ produce a $0.1 M_{\odot}$ object that entirely depletes its lithium content within about $10 \mathrm{Myr}$, whereas for the non accreting counterpart, complete lithium depletion takes more than 50 Myr. As clearly illustrated in Fig. 3, depending on the initial mass and the burst accretion rate, different episodic acccretion histories can produce objects with the same mass, say $0.1 M_{\odot}$, at a same age, say $\$ 50 \mathrm{Myr}$, exhibiting different levels of lithium depletion. Similar effects are found for episodic accretion sequences producing final objects within the entire characteristic domain of fully convective stars, $M \lesssim 0.35 M_{\odot}$.

\subsection{The case of stars that develop a radiative core}

If the central temperature exceeds $\sim 2-3 \times 10^{6} \mathrm{~K}$, a radiative core develops, because of the opacitiy decrease after the last " $\kappa$-bump" due to metals (C, O, Ne and Fe, see Rogers \& Iglesias 1992), as explained in Chabrier \& Baraffe (1997, see their Sect. 3.2 and their Fig. 9). The exact temperature at which this occurs depends on the density, given the sensitivity of the opacities to density in this temperature range (see Fig. 2a of Rogers \& Iglesias 1992). The higher the density, the higher the temperature required for the radiative core to develop. Since this range of temperatures is also characteristic of the temperature required for Li nuclear fusion, the central temperature and density at which the radiative core develops will determine the temperature at the bottom of the convective envelope and thus the level of $\mathrm{Li}$ depletion in the convective envelope. For sequences producing $1 M_{\odot}$ stars, Fig. 4 shows that accretion history has a strong impact on (i) the age for the onset of a radiative core; (ii) the mass of the convective envelope at ages $\$ 30 \mathrm{Myr}$; and (iii) the $\mathrm{Li}$ abundance in the convective envelope. The higher central temperature reached by sequences with strong burst accretion rates (see Fig. 2) results in a radiative core that develops earlier

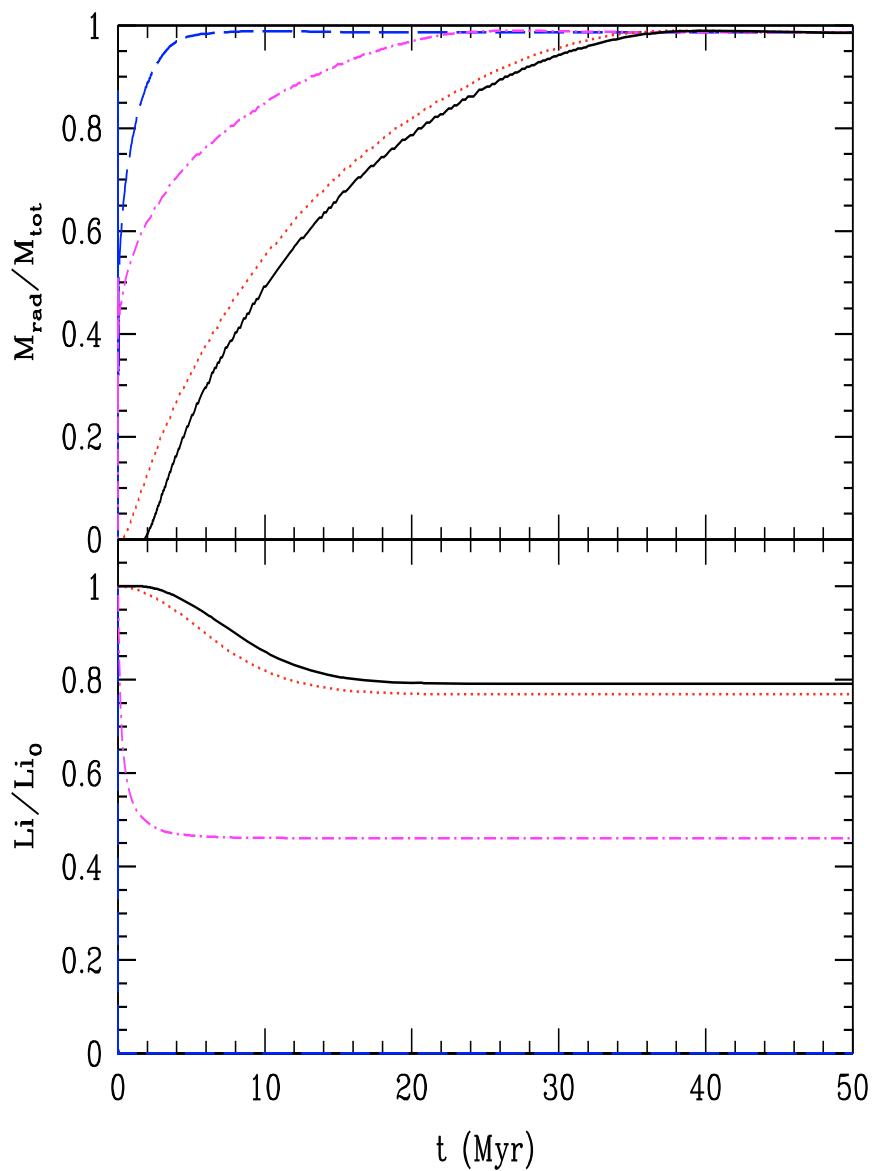

Fig. 4. Evolution of the mass of the radiative core divided by the total mass (upper panel) and of the surface lithium abundance divided by the initial Li abundance (lower panel) as a function of time for models producing a $1 M_{\odot}$ star. The curves refer to the same models as in Fig. 2. Note that $\mathrm{Li}$ is entirely depleted in the model with $M_{\text {init }}=10 M_{\text {Jup }}$ (long-dashed blue line) in less than $1 \mathrm{Myr}$.

in time. For the sequence starting from $M_{\text {init }}=10 M_{\mathrm{Jup}}$, its more compact structure yields significantly higher temperatures at the bottom of the convective envelope, with a maximum of $\sim 7 \times 10^{6} \mathrm{~K}$, resulting in complete Li depletion in the convective envelope at ages $<1$ Myr. In comparison, for the accreting sequence starting with $M_{\text {init }}=0.1 M_{\odot}$, the maximum temperature reached at the bottom of the convective envelope is $\sim 3.5 \times 10^{6} \mathrm{~K}$, which is comparable to the value found in the non accreting sequence (see Fig. 4). These differences in temperature are crucial for Li depletion, yielding very different final lithium abundances in the convective envelope, as illustrated in Fig. 4.

The main results of this section can be summarized as follows. Depending on the initial seed mass and the burst rates, different episodic accretion histories can produce $1 M_{\odot}$ star models with different sizes of the convective envelope at the same age, for ages $\$ 30$ Myr. The models can have different surface $\mathrm{Li}$ abundances even after the models have converged toward the same structure, i.e. after $30 \mathrm{Myr}$ in the particular case portrayed in Fig. 4. Similar effects are found for models with episodic accretion producing partly convective objects in the mass range $0.35-1 M_{\odot}$. 


\section{Discussion}

\subsection{Summary of the results}

We have shown in the present work that an early protostar/BD accretion history based on episodes of short, intense bursts of accretion with typical accretion rates $\dot{M}_{\text {burst }}=10^{-4}-5 \times$ $10^{-4} M_{\odot} \mathrm{yr}^{-1}$, as obtained in 2D hydrodynamical simulations of gravitationally unstable accretion disks (Vorobyov \& Basu 2005), can affect the internal structure of low-mass objects even after several Myr, up to a few times $10 \mathrm{Myr}$. The main results obtained in the present analysis can be summarized as follows.

(i) Episodic accretion produces objects with smaller radius and higher central temperature compared to the non accreting counterpart with the same mass at the same age.

(ii) Higher central temperatures at a given age and mass can significantly enhance lithium depletion in fully convective low-mass stars.

(iii) As a consequence of the hotter structure, a radiative core develops earlier in accreting objects with final masses $M \gtrsim 0.35 M_{\odot}$. Episodic accretion can thus lead to final objects with smaller convective envelopes than predicted by standard non accreting models, at ages up to a few times 10 Myr.

(iv) The more compact and hotter structure of accreting models with $M \gtrsim 0.35 M_{\odot}$ increases the maximum temperature reached at the bottom of the convective envelope, compared to the non accreting counterpart, increasing the level of lithium depletion in the convective envelope.

We stress that the magnitude of all these effects strongly depends on the initial mass, the acretion burst rate, and the total number of bursts, but barely depends on the phases of quiescent accretion, as long as $\dot{M}_{\text {quiet }} \ll 10^{-6} M_{\odot} \mathrm{yr}^{-1}$. Within the range of parameters mentioned in Sect. 2.2, episodic accretion can produce final low-mass star models that depart at various levels from standard non accreting models in terms of radius, luminosity (and thus position in the HRD, see BCG09), lithium abundance, and convective envelope mass, at a given similar age. Conversely, final objects issued from episodic accretion display observational signatures similar to non-accreting objects of same mass, but at a younger age, as shown in BCG09, the age difference again depending on the various accretion histories.

\subsection{Comparison to observations: anomalous lithium depletion in young clusters}

Several observational studies report discrepancies between ages inferred from evolutionary isochrones, on one hand, and from lithium depletion, on the other hand, for M to $\mathrm{G}$ type stars of various ages (e.g. Yee \& Jensen 2010). Observations also show lithium scatter in clusters and associations (e.g. da Silva et al. 2009; King et al. 2010) and young cluster members exhibiting severe, unexpected Li depletion for the inferred age of the cluster (e.g. Kenyon et al. 2005; Sacco et al. 2007). The present calculations provide a consistent solution to these puzzles, in particular providing an explanation for the anomalous lithium depletion observed in some members of clusters that are a few Myr old (Kenyon et al. 2005; Sacco et al. 2007, 2008; da Silva et al. 2009). The explanation suggested for these "interlopers", found for instance in $\sigma$ Ori, $\lambda$ Ori, and the ONC, is to invoke a significant age spread within these clusters. However, at least in some cases (see below), the required age spread is significant, up to $10-20 \mathrm{Myr}$, a rather unlikely possibility in such young clusters. The present calculations, based on early evolution sequences involving accretion outbursts, provide a more plausible explanation, as illustrated in Fig. 5 for the three weird objects discovered by Sacco et al. (2007) in $\sigma$ Orionis. These objects show severe Li depletion, inconsistent with the age of the cluster (about $5 \mathrm{Myr}$ ), but have radial velocities consistent with cluster membership. They are displayed in the upper panel of Fig. 5. Evolutionary sequences undergoing burst accretion with rates $\dot{M}_{\text {burst }} \sim 5 \times 10^{-4} M_{\odot} \mathrm{yr}^{-1}$ and reaching final masses $0.3 M_{\odot}$ and $0.7 M_{\odot}$, respectively, reach the observed locations in the $\mathrm{HRD}$ at an age of $\sim 5 \mathrm{Myr}$, consistent with the age of $\sigma$-Ori, whereas the same locations correspond to 10-15 Myr for the non accreting sequences of same mass, as shown in the figure. For the accreting sequence producing a $0.7 M_{\odot}$ object, the middle panel of Fig. 5 shows that a radiative core develops much earlier compared to the corresponding non accreting sequence. The lower panel illustrates the much faster Li depletion for the accreting sequences, as discussed in Sect. 3, with complete destruction occurring within less than $1 \mathrm{Myr}$ for the sequence producing a $0.7 M_{\odot}$ object. The possibility for the accretion burst models to explain consistently both the position in the HRD and the observed severe lithium depletion for these objects at the cluster age without particular fine tuning of the accretion parameters provides a strong support for this scenario. Although the high level of Li depletion in the three objects of Sacco et al. (2007) has recently been questioned by Caballero (2010), similar anomalous Li-depletions have been reported in other clusters by other groups (see references above), which can also be explained by episodic accretion. Our results thus reinforce the word of caution of da Silva et al. (2010) regarding the elimination of stars as cluster members based only on $\mathrm{Li}$ abundances. The present analysis demonstrates that lithium is not a reliable age indicator, as its fate strongly depends on the past accretion history experienced by the star!

The same episodic accretion calculations can explain the unexpected degree of lithium depletion of stars with mass $>0.5 M_{\odot}$ in the young cluster IC 4665 (Jeffries et al. 2009), or the spread in Li abundance in cool dwarfs of about a solar mass reported in the Pleiades (King et al. 2010), although other scenarios, based either on the star rotational history (Bouvier 2008) or stellar activity (King et al. 2010), have been suggested.

\subsection{Abundances in planet host stars}

Another interesting implication of our calculations and the inferred link between episodic accretion, lithium depletion, and the size of the convective envelope in young stars is the connection with the presence of close-in giant planets. Zhu et al. (2010b) suggest that the presence of mass clumps in some specific regions of circumstellar disks, which may lead eventually to non steady accretion when the disk becomes unstable (Vorobyov \& Basu 2005), could play an important role in the process of planet formation. Following up along these lines, we can speculate that the presence of giant planets, which require massive protoplanetary disks, more prone to gravitational instability, could be linked to the intensity of episodic accretion outburts. If this suggestion happens to be true, the present calculations provide an explanation for the claimed higher lithium depletion in planet host stars (Israelian et al. 2009; Sousa et al. 2010): since these planet host stars will have undergone more intense accretion bursts, they will experience more intense $\mathrm{Li}$ depletion than similar planetless stars whose presumably less massive disks are less prone to violent accretion-burst episodes. 

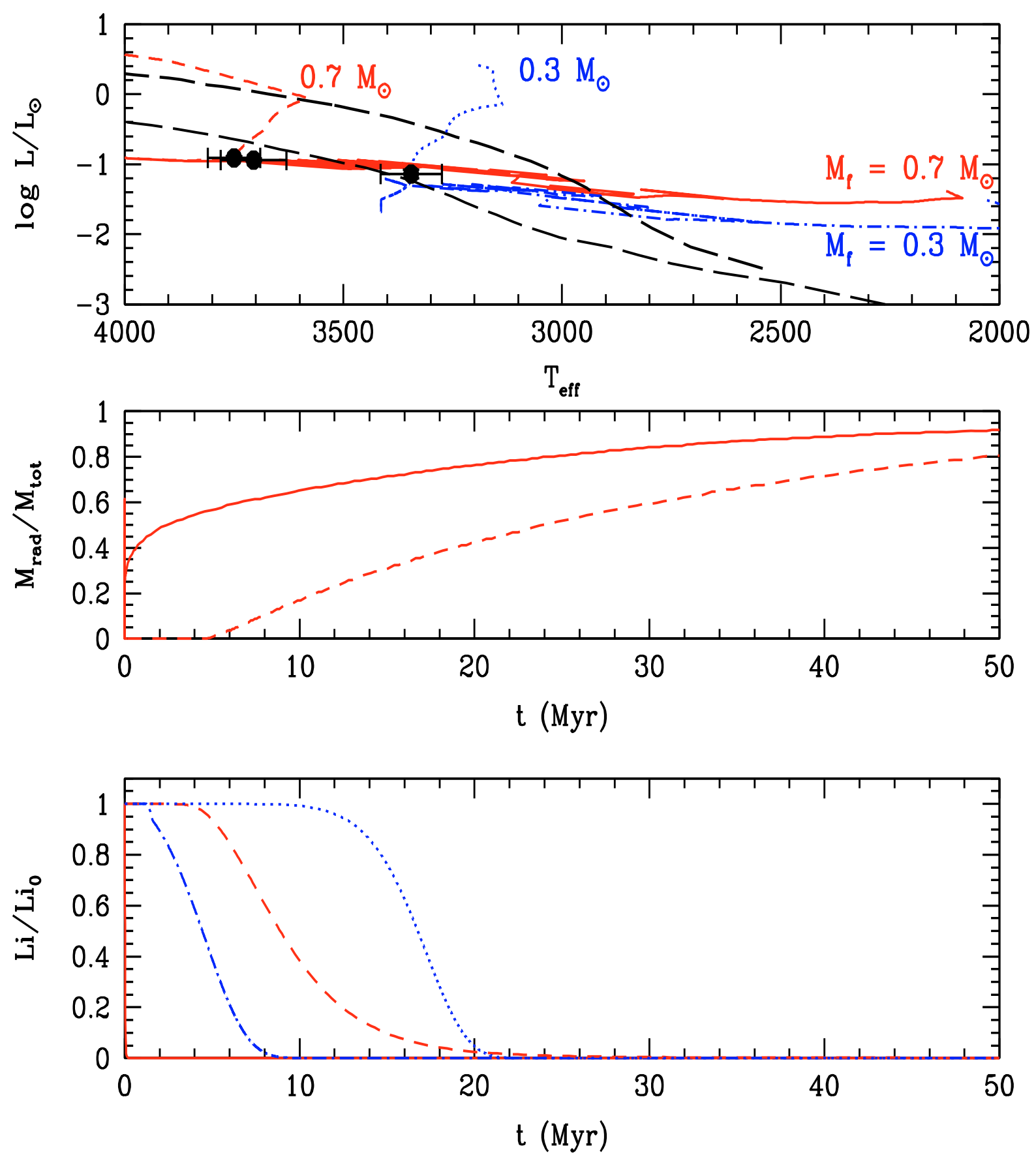

Fig. 5. Comparison of accreting models with observations of Sacco et al. (2007). Upper panel: HR diagram. The two long-dashed (black) curves are the $1 \mathrm{Myr}$ and $10 \mathrm{Myr}$ isochrones of Baraffe et al. (1998) for non accreting models. The black points are the objects of Sacco et al. (2007). Middle panel: radiative core mass versus time for models producing a $0.7 M_{\odot}$ star. Lower panel: surface Li abundance versus time. The solid red curve is indistinguishable from the $X$-axis (i.e. [Li] = 0): accreting sequence producing an object with final mass $M_{\mathrm{f}}=0.7 M_{\odot}$ with $M_{\text {init }}=20 M_{\text {Jup }}$, $N_{\text {burst }}=14$; dash-dotted blue: accreting sequence producing an object with final mass $M_{\mathrm{f}}=0.3 M_{\odot}$ with $M_{\text {init }}=10 M_{\text {Jup }}, N_{\text {burst }}=6$. In both cases, $\dot{M}_{\text {burst }}=5 \times 10^{-4} M_{\odot} \mathrm{yr}^{-1}, \Delta t_{\text {burst }}=100 \mathrm{yr}$ and $\Delta t_{\text {quiet }}=1000 \mathrm{yr}$; dashed red: non accreting $0.7 M_{\odot}$ model; dotted blue: non accreting $0.3 M_{\odot}$ model.

Furthermore, as shown in this paper, episodic accretion can produce objects whith smaller convective envelopes than the ones obtained with standard non accreting models at ages $\$ 10 \mathrm{Myr}$ (see Figs. 4 and 5), the characteristic maximum lifetime of protoplanetary disks. Consequently, we can expect the signature of (both terrestrial and gaseous) planet formation to leave a greater imprint on stars that have undergone phases of intense accretion bursts. Although speculative, this suggestion provides a consistent explanation for the recently claimed peculiar chemical abundance of the Sun, with about $20 \%$ depletion of refractory elements relative to volatile elements, compared with solar-type analogs harboring close-in giant planets (Melendez et al. 2009; Ramirez et al. 2009). According to these authors, this chemical peculiarity seems to be common among Sun-like stars with no detected giant planets and is said to reflect the signature of the formation of terrestrial planets, which trap the refractories, whereas the dust-depleted gas is accreted by the central star (Ramirez et al. 2009; Melendez et al. 2009). For such 
a suggestion to be viable, and the signature of terrestrial planet formation to be imprinted in the Sun photospheric composition, the Sun's convective envelope, of mass $M_{\mathrm{env}}$, must have been much smaller during the lifetime of the accretion disk, i.e. during the first $\sim 10 \mathrm{Myr}$, than predicted by standard pre-main sequence models. Indeed, let $X_{i}^{\prime}$ and $X_{i}$ be the mass fractions of element $i$ in the accreting material and in the originally unpolluted star convective envelope, respectively. The new abundance $X_{i}^{\prime \prime}$ of such element in the convective envelope becomes

$X_{i}^{\prime \prime}=\frac{X_{i}}{1+\alpha}\left(1+\alpha \frac{X_{i}^{\prime}}{X_{i}}\right)$,

where $\alpha=M_{\mathrm{acc}} / M_{\mathrm{env}}$ is the ratio of the accreted mass to the star convective envelope mass. The ratio of two elements, say $i \equiv$ refractories and $j \equiv$ volatiles, in the convective envelope after accretion of disk material becomes

$$
\frac{X_{i}^{\prime \prime}}{X_{j}^{\prime \prime}}=\frac{X_{i}}{X_{j}} \frac{1+\alpha \frac{X_{i}^{\prime}}{X_{i}}}{1+\alpha \frac{X_{j}^{\prime}}{X_{j}}} .
$$

In the presence of terrestrial planets, we expect $X_{i}^{\prime}<X_{i}$ and $X_{j}^{\prime}>X_{j}$, since most refractories remain trapped in the planet, and thus

$\frac{X_{i}^{\prime \prime}}{X_{j}^{\prime \prime}}<\frac{X_{i}}{X_{j}}$.

The larger $\alpha$, hence the smaller the convective envelope, the larger the inequality Eq. (7), hence the larger the indirect signature of planet formation in the star's convective envelope.

To obtain such a result, the aforementioned authors refer to the results of Wuchterl \& Klessen (2001), who explored the formation and early evolution of a $1 M_{\odot}$ star, and found that the early Sun was never fully convective. These calculations, however, are incorrect. First of all, as discussed in Baraffe et al. (2003), the calculations of Wuchterl \& Klessen (2001) yield an evolutionary track for a $1 M_{\odot}$ star that is much too hot, by $\sim 700-800 \mathrm{~K}$ in $T_{\text {eff }}$ at a given $L$, compared to observed young binary systems with (dynamically determined) masses around a solar mass. Second of all, the unrealistic assumption of spherical accretion in the Wuchterl \& Klessen calculations leads to significantly hotter inner structures, which will favor the growth of a radiative core, compared with more realistic 3D rotational collapse calculations (Chabrier et al. 2007, Fig. 3). This assumption is thus certainly responsible for the above-mentioned large overestimate of the effective temperature at young ages. On the other hand, the effect of episodic accretion on the mass of the convective envelope illustrated in Fig. 4 provides a more plausible explanation for this problem, and suggests that the early Sun could have undergone a phase of strong accreting bursts.

Although interesting, however, this interpretation of the peculiar refractory to volatile abundance ratio in the Sun and other solar twins without detected close-in giant planets as a consequence of terrestrial planet formation is challenged by the recent analysis of Chavero et al. (2010), based on four CoRoT planet host stars. This work suggests that alteration of abundances of refractories with respect to volatiles in stellar atmospheres may simply result from condensation processes in the accretion disk and from accretion of such altered gas onto the star. Independent of the source of this peculiar abundance pattern, the signature of such condensation/accretion processes will still leave a greater imprint if the star convective envelope is considerably smaller than usually expected for solar type stars at ages $<10$ Myr. Also, the analysis of Chavero et al. (2010) reveals no obvious correlation between abundances and condensation temperatures in the CoRoT stars, indicating no sign of overabundance of volatiles relative to refractories in stars harboring close-in giant planets compared to the Sun, contradicting the observations of Melendez et al. (2009) and Ramirez et al. (2009). The peculiar abundance ratio determinations in the Sun and solar analogs without detected giant planets must thus be confirmed by further studies.

In conclusion, anomalous abundance ratios of refractories to volatiles in stars may not necessarily reflect the signature of terrestrial planet formation but may also result from condensation processes in the disk. A detectable signature of these abundance anomalies, however, requires a smaller convective envelope during typical disk lifetimes than predicted by standard models. Strong accretion outburts do produce these favorable conditions.

\section{Conclusion}

We have shown here that non steady accretion can strongly affect the internal mechanical (radius) and thermal (temperature) structures, hence the observational signatures $\left(L, T_{\text {eff }}\right)$ of lowmass stars (and brown dwarfs), possibly up to ages of a few tens of Myr. The magnitude of the effects strongly depends on the forming object's initial core mass, the number of bursts, and the intensity of the burst accretion episodes. As already highlighted in BCG09, episodic accretion can produce models of a given final mass that to various extents depart from standard non accreting models in terms of position in the HRD, Li abundance, and convective envelope mass. Since the mechanisms responsible for the accreting disk formation and possible outbursts depend on many environmental parameters (e.g. mass, temperature, angular momentum, magnetic field of the parent collapsing cloud, and thermal properties of the disk), it is certainly possible to produce various populations of stars at a similar age in young clusters and associations that depart at various levels from predictions of standard pre-main sequence models. Therefore, our scenario does not systematically predict a broad spread among these stellar properties/signatures, because the level of departure entirely depends on the star or cluster properties and accretion histories. Further observational effort is needed to explore these issues in more detail and to try to have better constraints on the processes of non steady accretion during the early stages of evolution. Among the multiple consequences of early episodic accretion, we emphasize the limited (to say the least) reliability of Li depletion as an age or cluster membership indicator. We urge observers to re-analyze young cluster populations on the basis of the present results, since several genuine cluster members may have been eliminated because of their unexpectedly low $\mathrm{Li}$ abundance. Any information about the fraction of such Li depleted objects, with membership unambiguously assessed by radial velocity, could provide crucial insight into the occurrence and the intensity of non steady accretion.

We also suggest that intense bursts of episodic accretion may have some link to planet formation, if planet formation preferentially occurs in massive disks, which are more prone to instability. We speculate that stars harboring planets, including our Sun, may have experienced burst accretion episodes during their youth, which then led to a hotter thermal structure, thus to faster lithium depletion and a smaller outer convective zone than conventionally admitted. This provides a natural explanation for the higher lithium depletion observed in stars harboring at least giant planets. This also provides a plausible, although admittedly speculative, explanation for the claimed underabundance 
of refractory elements in the atmosphere of stars with no detected close-in giant planets, as a result of the formation of terrestrial planets, which trap large amounts of refractory elements. In any event, the multiple consequences of episodic accretion that we characterize in the present work should stimulate deeper theoretical and observational investigations, in order to better understand the twilight zone characteristic of (proto)star early evolution.

Acknowledgements. We are grateful to M. Asplund, J. Bouvier, L. Hartmann, A Morbidelli for valuable discussions. I.B. and G.C. thank the Max-Planck Institute for Astrophysics of Garching, where part of this work was completed, for their warm hospitality. This work was supported by the Constellation European network MRTN-CT-2006-035890, the French ANR "Magnetic Protostars and Planets" (MAPP) project, and the "Programme National de Physique Stellaire" (PNPS) of CNRS/INSU, France. The authors visited KITP, Santa Barbara, during completion of this work, and this research was supported in part by the National Science Foundation under Grant No. PHY05-51164.

\section{References}

Baraffe, I., Chabrier, G., Allard, F., \& Hauschildt, P. H. 1998, A\&A, 337, 403 Baraffe, I., Chabrier, G., Allard, F., \& Hauschildt, P. 2003, In Brown Dwarfs, IAU Symp., 211, 41

Baraffe, I., Chabrier, G., \& Gallardo, J. 2009, ApJ, 702, L27 (BCG09)

Bouvier, J. 2008, A\&A, 489, L53

Caballero, J. 2010, A\&A, 514, A18

Chabrier, G., \& Baraffe, I. 1997, A\&A, 327, 1039
Chabrier, G., Baraffe, I., Selsis, F., et al. 2007, Protostars and Planets V, ed. B. Reipurth, D. Jewitt, \& K. Keil, 623

Chavero, C., de la Reza, R., Domingos, R. C., et al. 2010, A\&A, 517, A40

da Silva, L., Torres, C., de la Reza, R., et al. 2009, A\&A, 508, 833

Enoch, M. L., Evans, N. J., Sargent, A. I., \& Glenn, J. 2009, ApJ, 692, 973

Gallardo, J. 2007, Ph.D. Thesis, Ecole Normale Supérieure de Lyon

Gallardo, J., Baraffe, I., \& Chabrier, G. 2009, Cool Stars 15, AIP Conf. Proc., 1094, 321

Hartmann, L., Cassen, P., \& Kenyon, S. J. 1997, ApJ, 475, 770

Israelian, G., Delgado, Mena, E., Santos, N., et al. 2009, Nature, 462, 189 Jeffries, R., Jackson, R., James, D., \& Cargile, P. 2009, MNRAS, 400, 317

King, J., Schuler, S., Hobbs, L., \& Pinsonneault, M. 2010, ApJ, 710, 1610 Kenyon, M. J., Jeffries, R. D., Naylor, T., et al. 2005, MNRAS, 356, 89 Melendez, J., Asplund, M., Gustafsson, B., \& Yong, D. 2009, ApJ, 704, L66 Ramirez, I., Melendez, J., \& Asplund, M. 2009, A\&A, 508, L17 Rogers, F., \& Iglesias, C. 1992, ApJS, 79, 507

Sacco, G., Randich, S., Franciosini, E., et al. 2007, A\&A, 462, L23

Sacco, G., Franciosini, E., Randich, S., \& Pallavicini, R. 2008, A\&A, 488, 167 Siess, L., \& Forestini, M. 1996, A\&A, 308, 472

Siess, L., Forestini, M., \& Bertout, C. 1997, A\&A, 326, 1001

Sousa, S., Fernandes, J., Israelian, G., \& Santos, N. 2010, A\&A, 512, L5 Stahler, S. W. 1988, ApJ, 332, 804

Stahler, S. W., \& Palla, F. 2005, The Formation of Stars (Wiley-VCH) Sugimoto, D., \& Nomoto, K. 1975, PASJ, 27, 197

Vorobyov, E. I. 2009, ApJ, 704, 715

Vorobyov, E. I., \& Basu S. 2005, ApJ, 633, L137

Wuchterl, G., \& Klessen, R. S. 2001, ApJ, 560, L185

Yee, J. C., \& Jensen, E. 2010, ApJ, 711, 303

Zhu, Z., Hartmann, L., Gammie, C., et al. 2010a, ApJ, 713, 1134

Zhu, Z., Hartmann, L., Gammie, C., et al. 2010b, ApJ, 713, 1143 